\title{
Measurement of Shielding Effectiveness of Cable and Shielding Configurations by Mode-Stirred Techniques
}

\author{
RAMON L. JESCH, SENIOR MEMBER, IEEE
}

\begin{abstract}
The shielding effectiveness of cable configurations having different shielding arrangements and of shielding configurations that are used to terminate cable shields for helicopter wiring were measured by mode-stirred techniques. The mode-stirred measurements were taken at discrete frequencies between $200 \mathrm{MHz}$ and $6 \mathrm{GHz}$. A description of the cable and shielding configurations is given along with plots of the measured shielding effectiveness data as a function of frequency.

Key Words-cables, connectors, mode-stirred chamber, relative leakage power, shielding effectiveness.

Index Code-F2b/f/g.
\end{abstract}

\section{INTRODUCTION}

$\mathrm{I}_{\mathrm{u}}^{\mathrm{N}}$ RECENT years, one major concern with the design and use of coaxial and multicontact connectors and accompanying cable assemblies has been the amount of electromagnetic (EM) energy or radio frequency (RF) leakage entering into the connector/cables that can be tolerated in today's advanced electronic systems. Moreover, these systems have to be designed to withstand the effects of electromagnetic interference (EMI) at operating frequencies from a few megahertz to many gigahertz. To facilitate system design, shielding effectiveness (SE) data are required for cables and connectors that interconnect various components, subassemblies, equipment, and subsystems. The evaluation of SE measurements of cables and connectors has been the subject of a great deal of analysis and research for some time.

To determine the SE of cable configurations having different shielding arrangements, the National Bureau of Standards (NBS), in 1985, undertook a program to conduct a study on standard practices of terminating cable shields for helicopter wiring for the U.S. Army Aviation Systems Command (AVSCOM). One of the objectives of this program was to determine a suitable technique for measuring the SE of terminated cable shields in order to compare the EMI effects between different cable arrangements. To accomplish this task, an extensive study of SE measurement techniques was conducted in the area of cables and connectors. Prominent among the measurement techniques being surveyed were the triaxial technique [1], which configures the test sample (cable/ connector under test) as a segment of transmission line, and

Manuscript received July 30, 1987; revised February 5, 1988. This work was supported by the U.S. Army Aviation Systems Command, St. Louis, MO 63120

The author is with the Electromagnetic Fields Division, National Bureau of Standards, Boulder, CO 80303.

IEEE Log Number 8821927 the mode-stirred technique [2], which uses a test chamber to expose the test sample to random incident fields. From the study taken [3], the mode-stirred technique was chosen as the most convenient technique to measure the SE of cables and connectors over a wide frequency range above a few hundred megahertz.

\section{Cable and Shielding Configurations}

\section{A. Cable Configurations}

Fig. 1 shows the four different cable configurations that were used to determine and compare the EMI effects of different shielding arrangements. The configurations were fabricated from $1.5 \mathrm{~m}$ (4.93 ft) lengths of $\mathrm{RG} / \mathrm{U}$ coaxial cable, which has two shields. The cable was modified to produce:

Configuration 1, consisting of the cable center conductor only, with the vinyl jacket and two shields removed from the cable;

Configuration 2, consisting of $\mathrm{RG} / \mathrm{U}$ cable retaining one shield only, with the other shield removed;

Configuration 3, consisting of the regular RG9/U cable with two shields;

and

Configuration 4, consisting of the regular RG9/U cable with one more shield added over the cable to give triple shielding.

All four cable configurations were equipped with Type $\mathrm{N}$ coaxial connectors.

\section{B. Shielding Configurations}

General types of shielding configurations that are used for helicopter wiring were discussed with AVSCOM and other knowledgeable personnel. It was decided to narrow down to three basic shielding configurations that should be measured for SE by NBS for the AVSCOM program. These included

1) Single Shielded Wire: The shield is stripped back approximately $15 \mathrm{~cm}$ (6 in) from one end of the wire and terminated by means of a solder sleeve and wire that is attached to the connector ground as shown in Fig. 2 .

2) Twisted Shielded Pair: The shield is terminated in the same manner as 1) above with one of the conductors attached 


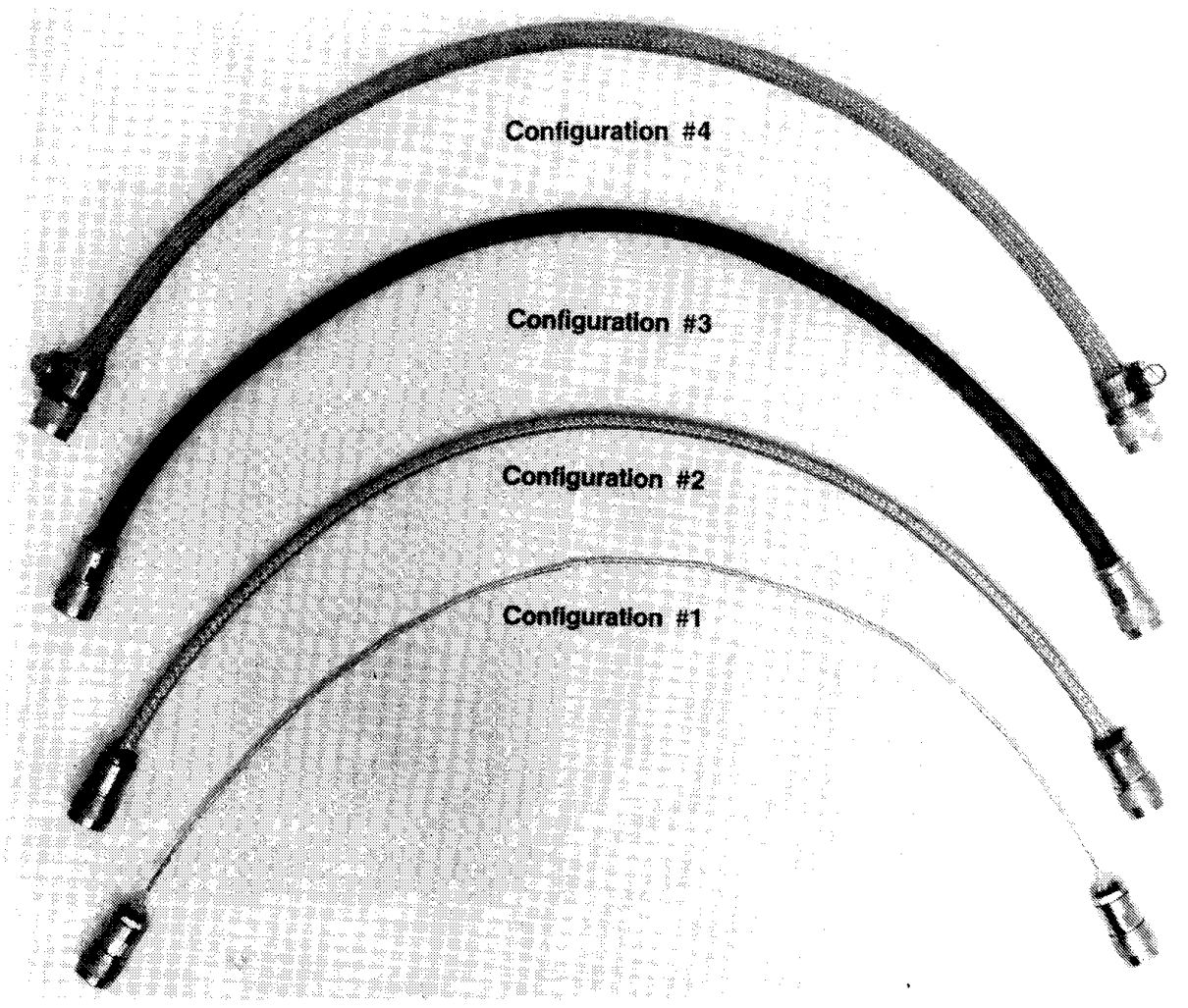

Fig. 1. Four different cable configurations.

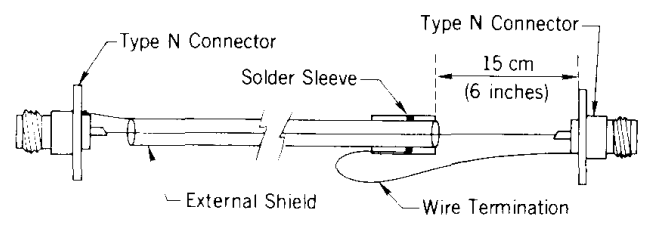

Fig. 2. Single shielded wire.

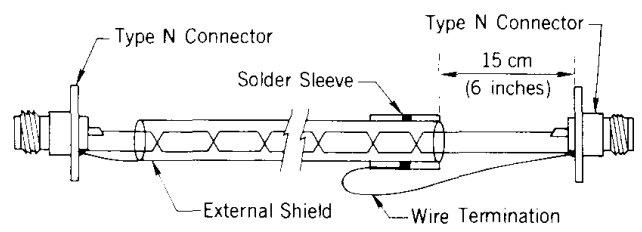

Fig. 3. Twisted shielded pair.

to the center conductor of the coaxial connector while the other conductor is attached to the connector ground as shown in Fig. 3.

3) Multipin Connector/Cable Arrangement: This arrangement is the most difficult to configure for SE measurements in the mode-stirred chamber. This involves interconnecting the shields together "daisy chains" and terminating the shields through a $50-\Omega$ connector in the chamber. Fig. 4 shows the cable arrangement of an actual harness assembly from an Army helicopter that was measured.

All three shielding configurations were equipped with Type $\mathrm{N}$ connectors.
III. Measurement Approach

\section{A. Mode-Stirred Technique}

The mode-stirred technique is a relatively new method for radiated susceptibility/vulnerability testing using a reverberation chamber. The idea of stirring or tuning the modes inside a large shielded enclosure (reverberation chamber) for electromagnetic compatibility (EMC) measurements was first proposed in 1968 [4]. The objective is to obtain a statistical, timeaveraged distribution of field strength or power density within a test volume inside the enclosure such that the location of the equipment or device under test (EUT) or (DUT) inside the chamber is not important.

The mode-stirred technique uses two operational approaches depending upon the voltage standing wave ratio (VSWR) for performing EMC measurements inside the chamber. The first approach, mode tuned (used when the input VSWR is relatively large in the order of 1.5 or more), steps the tuner at selected uniform increments (typically 200 steps or more), permitting measurements of the net input power, power received by the reference antenna, and the monitored DUT response at each tuner position. This allows corrections to be made for the variations in the chamber's test field resulting from changes in the VSWR of the transmitting antenna as a function of the tuner position. The second approach, mode stirred (used when the input VSWR is below 1.5), which was used in this paper for the SE measurements, rotates the tuner continuously while sampling the reference antenna received power and DUT response at rates much 


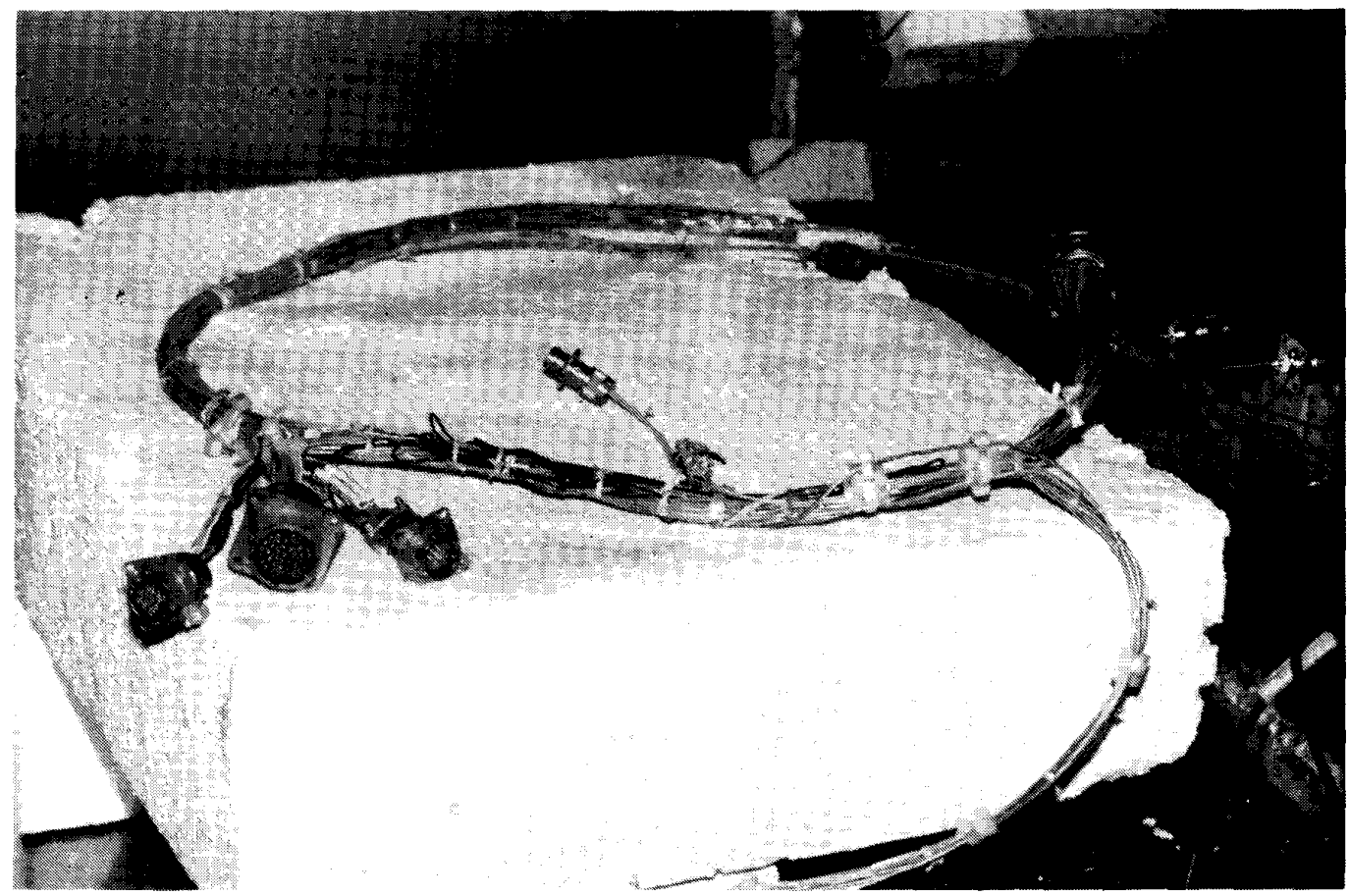

Fig. 4. Multipin connector/cable arrangement.

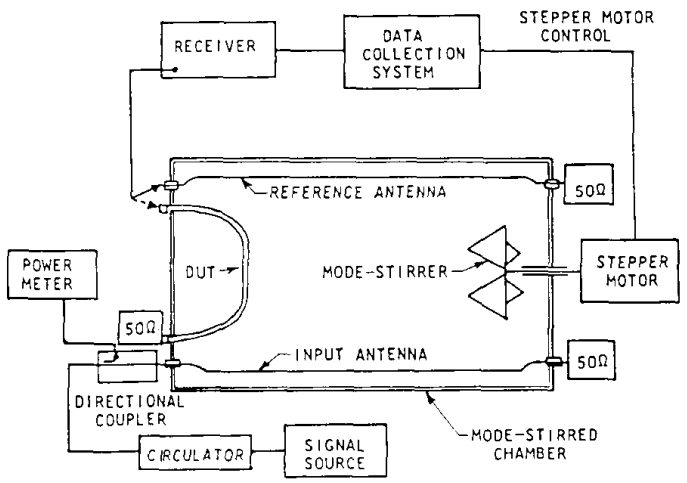

Fig. 5. Diagram of small mode-stirred chamber and test system.

faster than the tuner revolution rate. Large data samples up to 9999 can be obtained for a single tuner revolution, which takes approximately 3-6 min per revolution.

The mode-stirred technique for measuring the $\mathrm{SE}$ of connectors and cables was adapted by Bean and Hall [2] and is based upon a technique developed by Jarva [5] and work performed by others [6]. The military reported the use of the mode-stirred technique for measuring of the SE of connectors and cables in 1978 in proposed MIL-STD-1377A [7]. MILSTD-1344A [8] was later issued as a design guide of a modestirred test chamber and Method 3008 of the same standard for measuring the SE of multicontact connectors.

\section{B. Measurement System}

Two reverberation chambers different in size were used for the SE measurements between $200 \mathrm{MHz}$ and $6 \mathrm{GHz}$. Fig. 5 shows a block diagram of the small mode-stirred chamber at NBS with shielding configuration and test system for the frequency range between 1 and $6 \mathrm{GHz}$. Measurements of the $\mathrm{SE}$ of the cable and shielding configurations were made in this small chamber in accordance with Method 3008 of MIL-STD$1344 \mathrm{~A}$ [8] as required by MIL-C-38999H [9]. The wire antennas were later replaced with ridged-horn antennas and the measurements repeated. The small chamber, which has dimensions of $1.165 \mathrm{~m} \times 1.428 \mathrm{~m} \times 1.487 \mathrm{~m}(3.82 \mathrm{ft} \times$ $4.68 \mathrm{ft} \times 4.88 \mathrm{ft}$ ) is essentially a low-loss shielded enclosure that includes an input antenna, a reference antenna, and a mode stirrer. The antennas were placed in the chamber in such a way as to minimize direct coupling from the input (excitation) antenna to the reference (receiving) antenna and from the input antenna to the DUT. Testing is conducted inside the test chamber, whose smallest dimension is at least three wavelengths at the lowest test frequency. This is to assure an ample mode density, which is a necessary condition for the validity for the mode-stirred technique [10]. In addition, the minimum distance between the DUT and the chamber walls is at least one wavelength at the lowest test frequency to maintain a uniform electric field throughout the chamber. In order to insure that the shielding configurations (DUT) meet this criterion, each end of the DUT was connected directly to a short section of semi-rigid cable that extends inside the chamber as shown in Fig. 6. The semi-rigid cables in turn were connected to the bulkhead connectors on the chamber wall.

Fig. 7 is a block diagram of the larger mode-stirred reverberation chamber and measurement system that was used for the SE measurements between $200 \mathrm{MHz}$ and $1 \mathrm{GHz}$ [11]. 


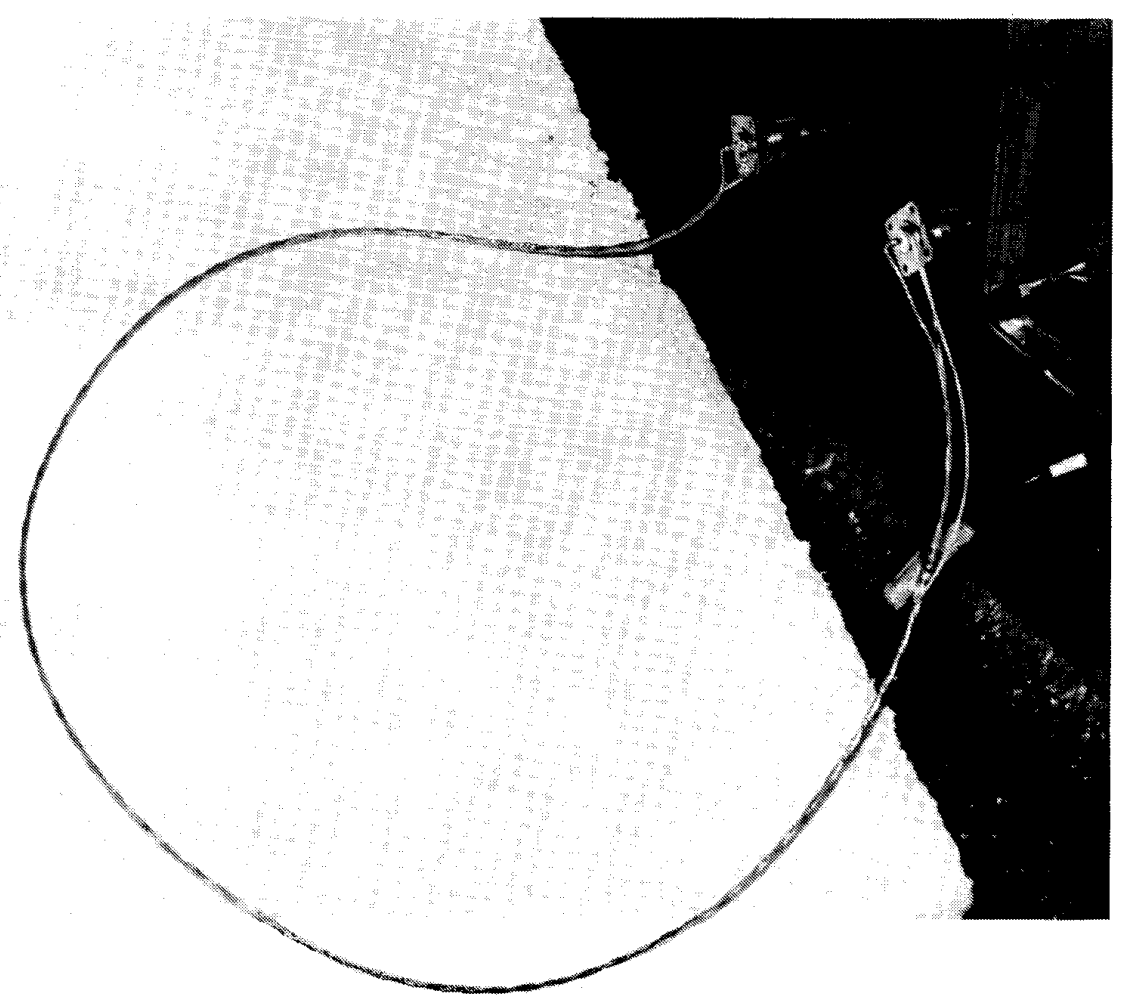

Fig. 6. DUT connected to semi-rigid cables inside the chamber.

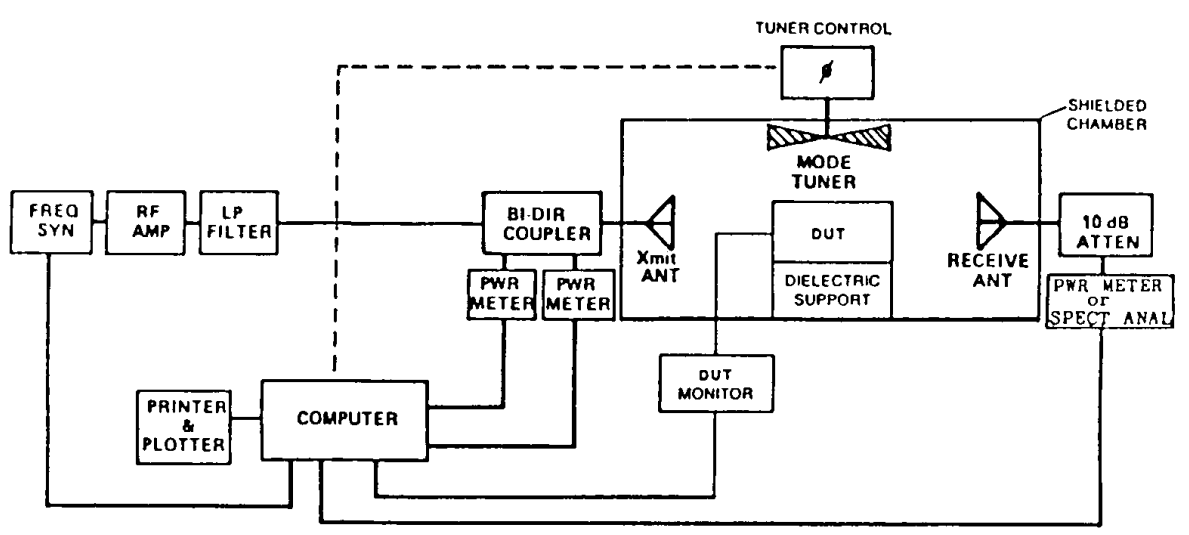

Fig. 7. Block diagram of the large mode-stirred chamber and measurement system.

The dimensions of the chamber are $2.74 \mathrm{~m} \times 3.05 \mathrm{~m} \times 4.57$ $\mathrm{m}(8.99 \mathrm{ft} \times 10 \mathrm{ft} \times 15 \mathrm{ft})$, which allow a lower frequency of approximately $200 \mathrm{MHz}$.

The test field in either chamber is established by means of RF source(s) connected to the input (transmitting) antenna(s) placed inside the chamber. Two different types of antennas are required to cover the frequency range between $200 \mathrm{MHz}$ and 6 $\mathrm{GHz}$, which include:

1) $\log$ periodic $(200$ and $1000 \mathrm{MHz})$ for the large chamber,

2) Ridged horn ( 1 and $6 \mathrm{GHz}$ ) for the small chamber.
Fig. 8 shows the placement of the DUT and the log periodic antennas inside the large mode-stirred chamber. The DUT consists of the single shielded wire shielding configuration that is connected directly to the short sections of semi-rigid cable that extends inside the chamber.

\section{Shielding Effectiveness}

The relative leakage power for determining SE is the RF power, which leaks into a cable, connector, or other device at a given frequency and is defined by the relationship

$$
\mathrm{SE}=10 \log _{10}\left(P_{1} / P_{2}\right) \mathrm{dB}
$$


226

IEEE TRANSACTIONS ON ELECTROMAGNETIC COMPATIBILITY, VOL. 30, NO. 3, AUGUST 1988

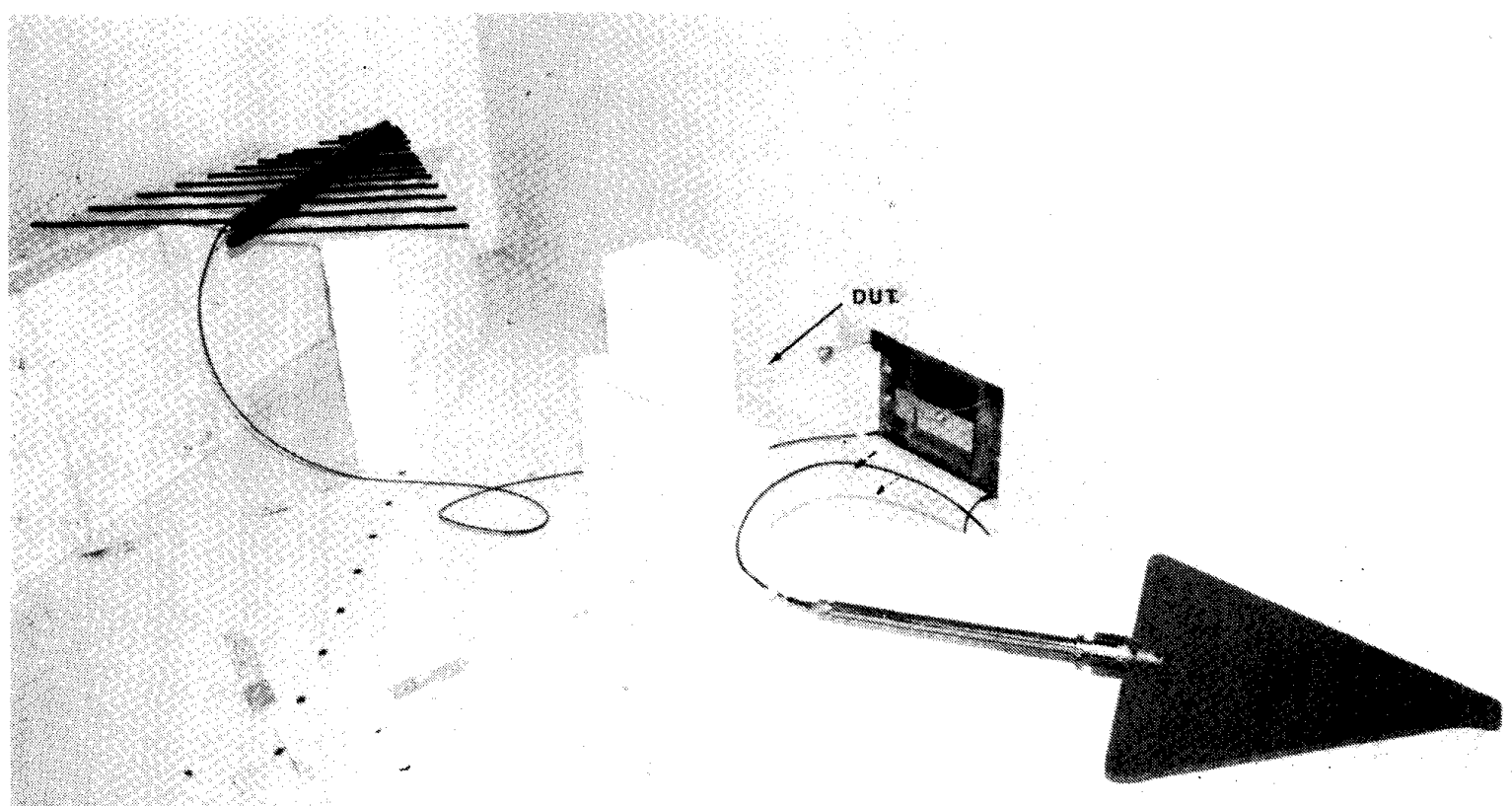

Fig. 8. Placement of the DUT and the log periodic antennas inside the large mode-stirred chamber.

where

$P_{1}$ is the total power received by the reference antenna for a given power applied to the input antenna, and

$P_{2}$ is the total power leaked into the DUT for the same power applied to the input antenna.

To measure the SE of the cable or shielding configurations (DUT) in the mode-stirred chamber, reference conditions are established with the DUT inside the chamber. Energy is injected inside the chamber from a source into the input (excitation) antenna to set up an electric field. The electric field in turn is received and coupled into the reference (receiving) antenna to establish a reference level which is measured as $P_{1}$ by the detection system. The objective is to obtain a time average for $P_{1}$ due to variations of field strength or power density inside the chamber. Next with the input antenna receiving the same amount of power as before, the detection system is disconnected from the reference antenna and connected directly to the DUT. The output power leaking into the DUT is measured as $P_{2}$. The SE of the DUT is determined using (1) by taking the ratio of the averages $\left(P_{1}\right.$ and $P_{2}$ ) over one rotation of the mode stirrer.

IV. Measurement Results

A. Cable Configurations

Figs. 9-12 show SE data that were obtained on cable configurations $1,2,3$, and 4 between $600 \mathrm{MHz}$ and $6 \mathrm{GHz}$ using the small mode-stirred chamber. These data were obtained to establish reference conditions with the cable configurations to use as a benchmark for comparing the EMI effectiveness of the AVSCOM shielding configurations. The $\mathrm{SE}$ varies considerably between the different cable configure-

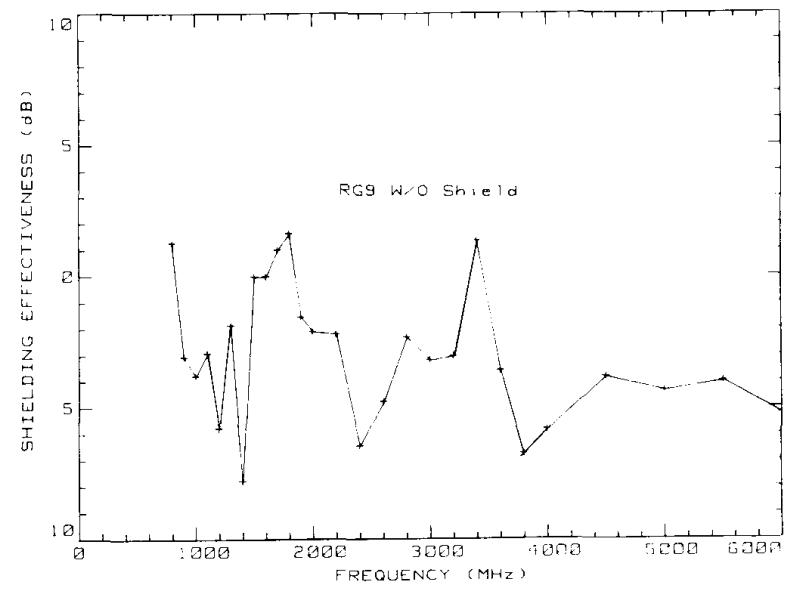

Fig. 9. SE of configuration 1 at selected frequencies between 600 and 6000 $\mathrm{MHz}$ using the small mode-stirred chamber with ridged-horn antennas.

ions. For example, the difference in $\mathrm{SE}$ between cable configurations 1 and 4 is approximately $100 \mathrm{~dB}$ at $1 \mathrm{GHz}$ while the difference between configurations 3 and 4 is about $20 \mathrm{~dB}$.

B. Shielding Configurations

Figs. 13-16 show SE data that were obtained on the shielding configurations 1,2 , and 3 for the AVSCOM program between $200 \mathrm{MHz}$ and $6 \mathrm{GHz}$ using the mode-stirred chambers. The large mode-stirred chamber was used for the SE measurements between $200 \mathrm{MHz}$ and $1 \mathrm{GHz}$ while the small mode-stirred chamber was used for the SE measuremints between 1 and $6 \mathrm{GHz}$. On configuration 3 (multipin connector/cable arrangement), two separate SE measurements were made with the harness assembly, which included 


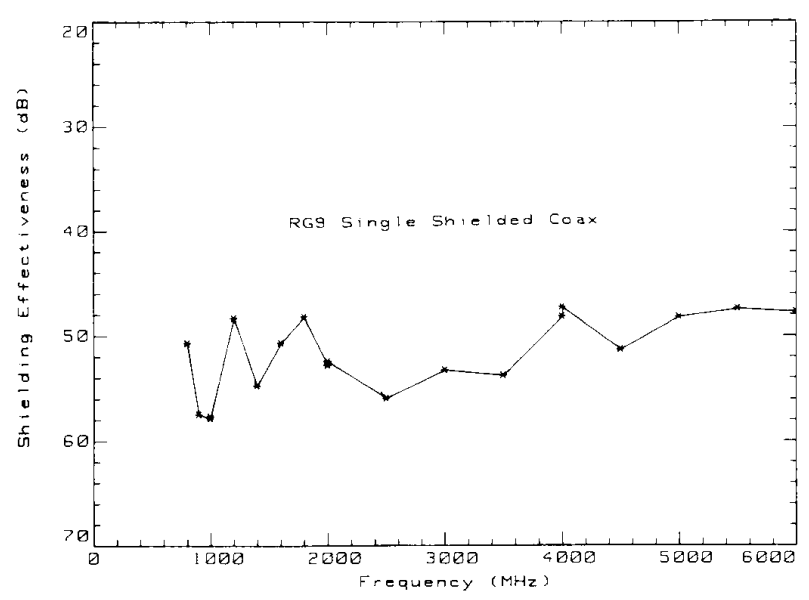

Fig. 10. SE of configuration 2 at selected frequencies between 600 and $6000 \mathrm{MHz}$ using the small mode-stirred chamber.

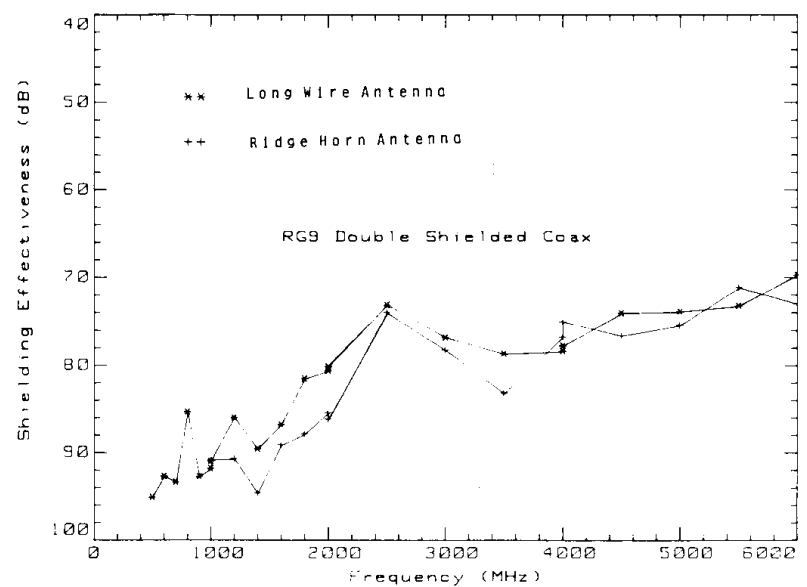

Fig. 11. SE of configuration 3 at selected frequencies between 600 and $6000 \mathrm{MHz}$ using the small mode-stirred chamber with both the long-wire and ridged-horn antennas.

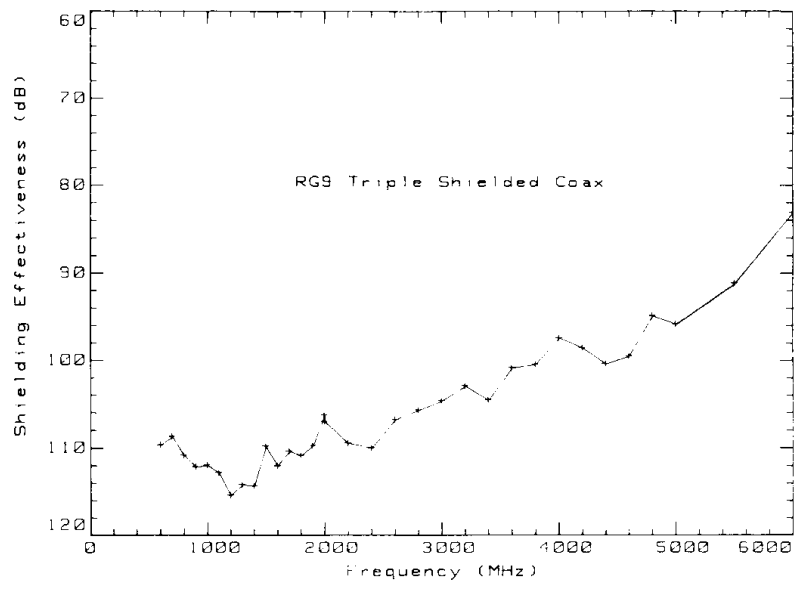

Fig. 12. SE of configuration 4 at selected frequencies between 600 and $6000 \mathrm{MHz}$ using the small mode-stirred chamber.

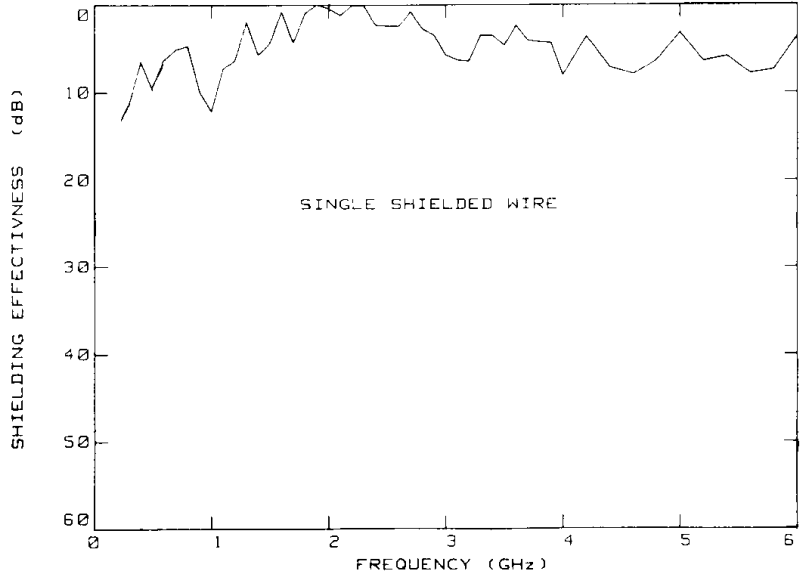

Fig. 13. SE of shielding configuration 1 at selected frequencies between $200 \mathrm{MHz}$ and $6 \mathrm{GHz}$ using the mode-stirred chambers.

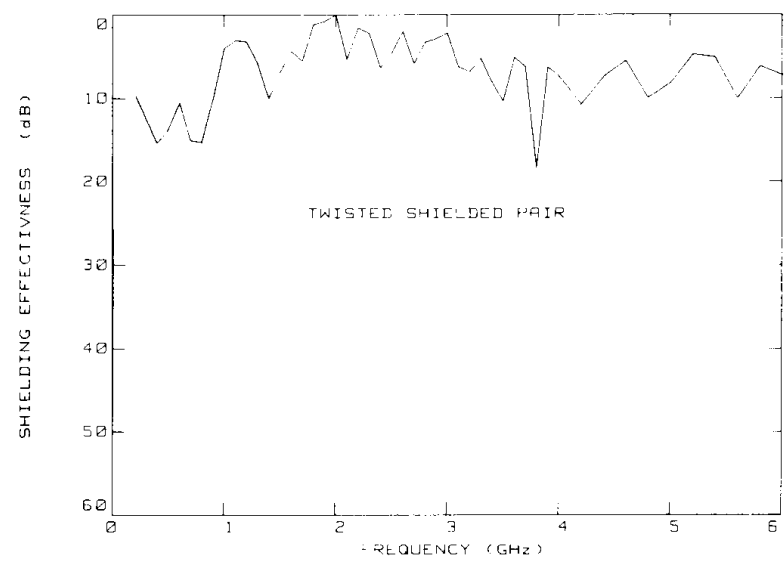

Fig. 14. SE of shielding configuration 2 at selected frequencies between $200 \mathrm{MHz}$ and $6 \mathrm{GHz}$ using the mode-stirred chambers.

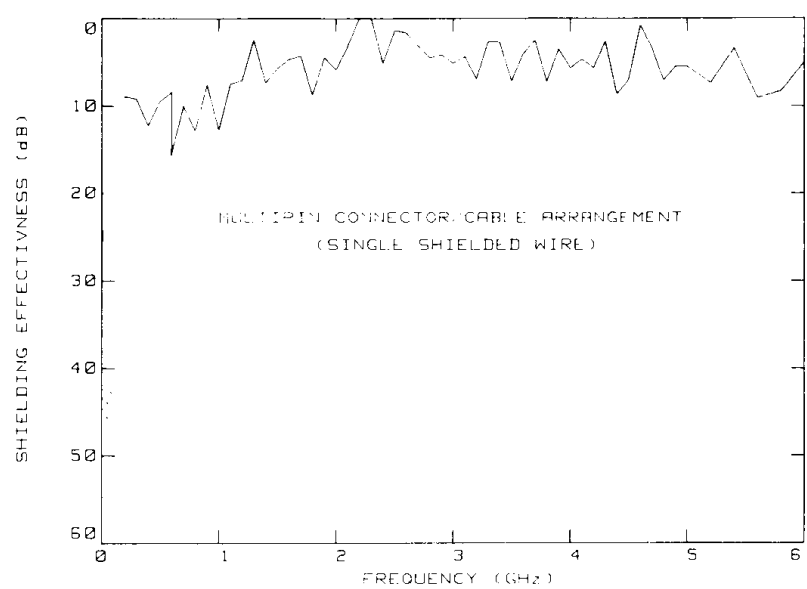

Fig. 15. SE of shielding configuration 3 with single shielded wire at selected frequencies between $200 \mathrm{MHz}$ and $6 \mathrm{GHz}$ using the mode-stirred chambers. 


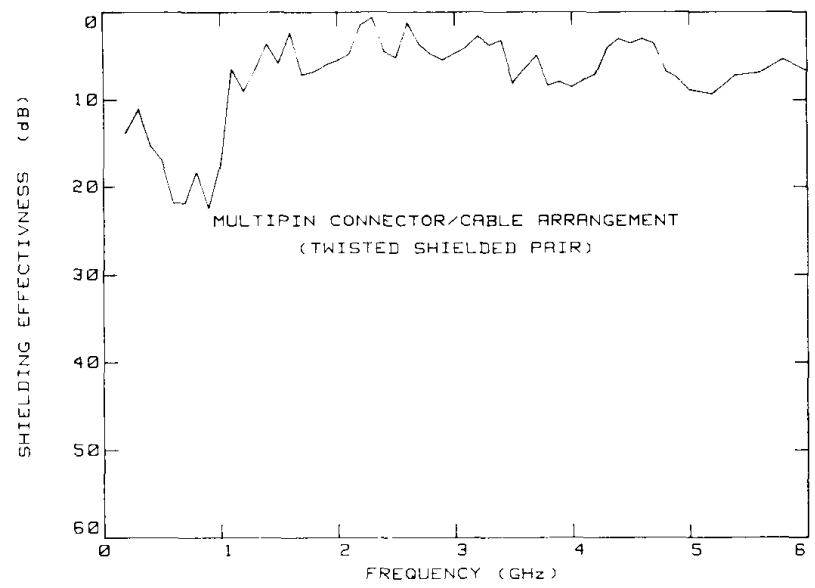

Fig. 16. SE of shielding configuration 3 with twisted shielded pair at selected frequencies between $200 \mathrm{MHz}$ and $6 \mathrm{GHz}$ using the mode-stirred chambers.

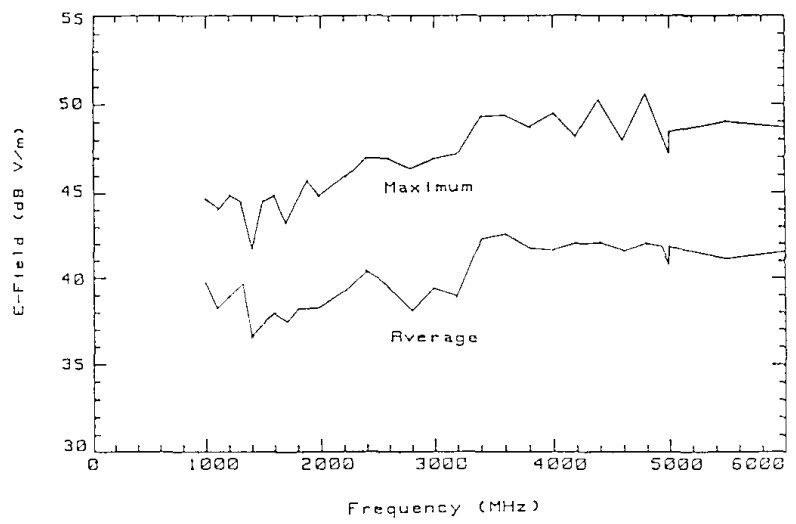

Fig. 17. Average and maximum E-field strength inside small NBS modestirred chamber for $1-\mathrm{W}$ net input power.

investigating the SE of the single shielded wire in the harness and the twisted shielded wire in the harness.

Overall, the configurations offer very little shielding from EMI. It appears that the twisted shielded pair offers a bit more shielding $(\sim 2 \mathrm{~dB}$ at several frequencies) than the single shielded wire.

\section{Measurement Uncertainty}

The estimated measurement uncertainties for SE of all cable and shielding configurations in the mode-stirred chamber is $\leq$ $\pm 4 \mathrm{~dB}[11]$.

Fig. 17 shows the average and maximum E-field strength inside the small NBS mode-stirred chamber for $1-\mathrm{W}$ net input power. The maximum and average electric field strengths inside the small NBS chamber were determined from the receiving antenna power measurements [11]. These data were obtained for a $1-W$ net input power to the chamber's transmitting antenna. Similar field strength information for the large NBS mode-stirred chamber is contained in [11].

\section{Summary and Conclusion}

Plots of SE were obtained for cable and shielding configurations which allowed the EMI effectiveness of the shielding configurations for the AVSCOM program to be compared with the reference conditions of the cable configurations. The SE measurements of the cable and shielding configurations were measured by mode-stirred techniques. This technique is ideal for measuring cables and multipin connector/cable arrangements over a wide frequency range. Different diameter sizes of cables and connectors can be conveniently measured in the same chamber. Moreover, high-level fields can be generated efficiently around the DUT with no requirement to rotate the DUT.

Shields terminated by means of a solder sleeve and wire, daisy chains, and other noncontinuous shielding arrangements do not provide adequate shielding from the surrounding EMI and they allow electronic systems to be exposed to high-level fields. Even at $200 \mathrm{MHz}$, these shielding configurations offer little protection from EMI. Other measurements of SE on other shielding arrangements and devices need to be determined and the results compared.

\section{ACKNOWLEDGMENT}

The author wishes to thank M. Crawford for helpful discussions on measurement techniques and data analysis, and J. Workman for his role in preparing the cable and shielding configurations, and making the measurements.

\section{REFERENCES}

[1] J. Zorzy and R. F. Muehlberger, "RF leakage characteristics of popular coaxial cables and connectors, $500 \mathrm{Mc}$ to $7.5 \mathrm{Gc}$," Microwave $J$, vol. 4, pp. 80-86, Nov. 1961.

[2] J. L. Bean and R. A. Hall, "Electromagnetic susceptibility measurements using a mode-stirred chamber," in Proc. IEEE Int. EMC Symp. (Atlanta, GA), pp. 143-150, June 20-22, 1978.

[3] R. L. Jesch, "A survey of triaxial and mode-stirred techniques for measuring the shielding effectiveness of connectors and cables," Nat. Bur. Stand. (U.S.) NBSIR 86-3060, Oct. 1986.

[4] H. A. Menes, "A new approach to electromagnetic field-strength measurements in shielded enclosures," in Wescon Tech. Papers (Los Angeles, CA), Aug. 1968.

[5] W. Jarva, "Shielding tests for cables and small enclosures in the 1- to 10-GHz range," IEEE Trans. Electromagn. Compat., vol. EMC-12, no. 1, pp. 12-24, Feb. 1970.

[6] "Characterization of shielding effectiveness measurement technique," McDonnell Douglas Astronautics Co., St. Louis, MO, Final Rep. MDCE1516, 1976.

[7] Proposed MIL-STD-1377A (Navy), "Shielding effectiveness of cables, connectors, and enclosures, measurement of." Oct. 1978.

[8] MIL-STD-1344A, "Appendix A, design of mode-stirred test chamber. Method 3008. Shielding effectiveness of multicontact connectors." Sept. 1980.

[9] MIL-C-38999H, “Military specification, connectors, electrical, circu lar, miniature, high density, quick disconnect, environment resistant, removable crimp contacts, and hermetic contacts." Feb. 1981.

[10] M. T. Ma, M. Kanda, M. L. Crawford, and E. B. Larsen, "A review of electromagnetic compatibility/interference measurement methodologies," Proc. IEEE, vol. 73, no. 3, pp. 388-411. Mar. 1985.

[11] M. L. Crawford and G. H. Koepke, "Design, evaluation, and use of a reverberation chamber for performing electromagnetic susceptibility/ vulnerability measurements," Nat. Bur. Stand., Tech. Note 1092. Apr. 1986. 